\title{
INCORPORACIÓN DE LA REVOCATORIA DE MANDATO EN LAS \\ CONSTITUCIONES DE AMÉRICA LATINA: ¿AMPLIANDO LA DEMOCRACIA DIRECTA O REFORZANDO EL PRESIDENCIALISMO?
}

\author{
INCORPORATION OF THE RECALL IN THE CONSTITUTIONS OF LATIN
}

AMERICA: EXPANDING DIRECT DEMOCRACY OR REINFORCING

PRESIDENTIALISM?

María Laura Eberhardt ${ }^{1}$

\begin{abstract}
RESUMO: Desde los años 80, y en un contexto de crisis de la representación, varias constituciones latinoamericanas adoptaron instrumentos participativos a fin de oxigenar las desgastadas instituciones de la democracia indirecta y acercar la toma de decisiones a los ciudadanos. Entre ellos destaca la revocatoria de mandato, en tanto habilita la destitución anticipada de los gobernantes a través del voto ciudadano. La incorporación constitucional de este mecanismo pone a la soberanía popular por encima de los representantes. Sin embargo, dicho avance participativo y democrático encubre, muchas veces, una estrategia política opuesta, destinada a aumentar y reforzar la concentración de facultades en el Ejecutivo. Este artículo analiza el proceso de incorporación de la revocatoria de mandato en las constituciones de Venezuela, Bolivia, Ecuador y Perú en el periodo 1993-2009 a fin de poner de manifiesto su real función e impacto participativo en el contexto de las reformas de las que formaron parte.
\end{abstract}

Palavras-chave: Constituciones latinoamericanas; Crisis de la representación; Hiperpresidencialismo; Mecanismos de participación y control ciudadano; Revocatoria de mandato

ABSTRACT: Since the 1980s, and in a context of crisis of representation, several Latin American constitutions adopted participatory instruments in order to oxygenate the worn out institutions of indirect democracy and bring decision-making closer to citizens. Among them, the revocation of the mandate stands out, as it enables the early dismissal of the rulers through the citizen vote. The constitutional incorporation of this mechanism puts popular sovereignty above the representatives. However, this participatory and democratic advance conceals an opposite political strategy, aimed at increasing and reinforcing the concentration of powers in the Executive. This article analyzes the process of incorporation of the revocation of mandate in the constitutions of Venezuela, Bolivia, Ecuador and Peru in the period 1993-2009, in order to show its real role and participatory impact in the context of the reforms of which they were part.

1 Doctora en Ciencia Política (UNSAM), Doctora por la Facultad de Derecho (UBA), Posdoctora por la Facultad de Derecho (UBA), Magíster en Ciencia Política y Sociología (FLACSO), Licenciada en Ciencia Política (UBA), Investgadora del CONCET, Profesora de UBA y UNAJ. ORCID: http://orcid.org/0000-00034447-5641.E-mail: laura_rafaela@yahoo.com.ar. 
Keywords: Latin American Constitutions; Crisis of Representation; Hyperpresidencialism; Participation and Control Mechanisms; Recall.

Sumário: 1. Introducción; 2. Algunos conceptos teóricos básicos; 3. Incorporación de la revocatoria de mandato en las constituciones latinoamericanas; a. Venezuela; b. Bolivia; c. Ecuador; d. Perú; 4. Consideraciones finales; 5. Referencias.

\section{INTRODUCCIÓN}

Avanzada la década de 1980, gran parte del mundo occidental atravesaba una crisis de representación o clima general de descontento popular respecto del desempeño de las instituciones democráticas y de los gobernantes (EBERHARDT, 2013, s.p.). En este contexto, los parlamentos se vieron desprestigiados como lugares donde reinaba "la politiquería y la verbosidad" (NINO, 1997, p. 237-238), con lobbistas operando detrás de la escena y con un funcionamiento permanentemente ineficiente y lento; al tiempo que los partidos políticos eran concebidos como meros grupos corporativos abocados a la defensa de sus intereses.

América Latina no fue ajena a esta realidad. En todos los casos (aunque en diferente grado), hubo cierta descomposición del Estado junto con la deslegitimación de los representantes (LISSIDINI, 2008, p. 127). Los partidos perdieron centralidad, cuando no han colapsado, y surgieron outsiders que prometieron más participación ciudadana directa a costa del Poder Legislativo (como Chávez en Venezuela y Correa en Ecuador).

Varias constituciones y legislaciones nacionales y locales fueron objeto de reformas políticas que, entre otras innovaciones, incorporaron una diversidad de mecanismos de participación y control ciudadano destinados a complementar las instituciones representativas con una intervención más directa de la ciudadanía en las decisiones públicas y en el monitoreo de los mandatarios. Dicha incorporación fue especialmente prolífica en el ámbito subnacional, donde las innovaciones en participación y el involucramiento de las entidades sociales contribuyeron a revitalizar la democracia local (BUCEK; SMITH, 2000, 3).

Entre tales mecanismos, destaca la revocatoria de mandato, cuya adopción será analizada en particular en este trabajo. Ello, debido a que se trata de un instituto de democracia directa (el voto de la gente es el que decide) que, junto con la participación ciudadana (expresar una opinión en un referéndum), también implica una alta dosis de control vertical o rendición de cuentas, ya que se emplea para separar anticipadamente del cargo a un representante electo que ha defraudado las expectativas de la población. 
Su importancia, y el motivo del interés que nos suscita, se debe a que su incorporación en las constituciones nacionales puede entenderse, en principio, como indicador de una contundente apuesta a la democracia participativa, ya que mantiene a la voluntad popular soberana por encima del poder de los representantes durante la mayor parte de su período. Reinterpretando a Locke podríamos decir que la revocatoria de mandato permite al pueblo recuperar anticipadamente el poder de decisión que delegó en forma temporal en sus mandatarios para depositarlo en nuevas manos. A diferencia del voto electivo, este instrumento permite desbancar a un gobernante durante el ejercicio de su cargo, sin tener que esperar a que caduque su mandato.

En América Latina, seis países la incluyeron en sus Constituciones nacionales: Venezuela, Bolivia, Ecuador y Perú, Colombia y Panamá. En los tres primeros casos, la revocatoria alcanza incluso a la figura del presidente, convirtiéndose, al menos en apariencia, en una institución llamada a modificar el funcionamiento tradicional del presidencialismo (al flexibilizar el mandato fijo de los mandatarios electos).

No obstante, la adopción constitucional de la revocatoria de mandato, junto con la de otros diversos mecanismos de participación ciudadana, tuvo como base o contracara en numerosos casos un simultáneo acrecentamiento y concentración de facultades en el presidente (impulsores unilaterales de tales reformas estructurales), que pusieron en jaque la capacidad real de ejercicio de un control horizontal por parte de los poderes Legislativo, Judicial y/o federales, así como de la accountability vertical por parte de la población.

Es decir, que no en pocas ocasiones, la adopción constitucional de instrumentos semidirectos y/o directos como el recall $^{2}$ tuvo en realidad como función enmascarar o maquillar otras reformas de fondo orientadas a desequilibrar la estructura de poderes horizontal y vertical a favor del Ejecutivo. De este modo, al tiempo que se aumentaban los derechos potenciales de los ciudadanos a participar y controlar a sus gobernantes, se fortalecía y ampliaba el poder unipersonal del presidente, el que en adelante gozaría de una capacidad suficiente para neutralizar y/o anular todo ejercicio de tales controles y derechos.

El objetivo del presente trabajo consiste en analizar el proceso de incorporación constitucional de la revocatoria de mandato en Venezuela, Bolivia, Ecuador y Perú. Ello, en el

2Revocatoria de mandato en inglés. 
contexto de una crisis de la representación política que dio el marco para acrecentar la participación ciudadana, así como de reformas de la estructura de poder que implicaron la ampliación y concentración de facultades en el presidente. Se busca poner en evidencia las características y ambigüedades de este proceso, que pudo implicar tanto un avance en términos participativos y democráticos (la adopción de contundentes vías de participación y control vertical como la revocatoria), como un retroceso en términos republicanos y liberales (el simultáneo acrecentamiento, concentración y desequilibrio de poderes a favor del presidente).

\section{ALGUNOS CONCEPTOS TEÓRICOS BÁSICOS}

En este trabajo se analizará el proceso de incorporación de la revocatoria de mandato en las constituciones latinoamericanas desde los años 80. El interrogante que subyace es si la adopción de este y otros mecanismos de participación y control ciudadano se realizó en el marco de una reforma tendiente a democratizar el régimen, dando un espacio real al involucramiento cívico y empoderando a la población o si, por el contrario, constituyó una especie de maquillaje o distractivo respecto de otras reformas de fondo orientadas a aumentar y concentrar las facultades en el Ejecutivo en detrimento de los otros poderes, tanto horizontales (Legislativo y Judicial) como verticales (los gobiernos subnacionales).

En el segundo caso, el desequilibrio de potestades a favor del órgano unipersonal de la presidencia es un factor que pone en riesgo el ejercicio efectivo de cualquier forma de control o accountability sobre éste: tanto horizontal (por los otros poderes: el Congreso, la Justicia y/o los gobiernos subnacionales) como vertical (por la ciudadanía). En tal sentido, la sobrecarga de atributos en el Ejecutivo puede invalidar, anular o neutralizar la utilización efectiva de las nuevas herramientas de democracia directa, abonando a un régimen hiperpresidencialista que pone en riesgo la vigencia de un régimen liberal republicano.

Dicho esto cabe definir brevemente los conceptos antes mencionados. Para comenzar, hemos caracterizado a la revocatoria de mandato como un "mecanismo de democracia directa". Estos mecanismos permiten a los ciudadanos decidir sobre materias específicas directamente en las urnas. Son instrumentos de decisión política ejercidos a través del sufragio directo y universal (ALTMAN, 2005, 204). Su finalidad es hacer que los ciudadanos participen colectiva y directamente en el procedimiento de toma de decisión, más que para elegir a sus representantes, para tomar sus propias decisiones (PAYNE et al, 2002, s.p.)

En cuanto a la "revocatoria de mandato", es un instituto que habilita a los ciudadanos a dejar sin efecto el mandato de aquellas personas elegidas por voto popular que han dejado de 
contar con su aprobación y confianza. Somete a los funcionarios a la rendición de cuentas (CRONIN, 1989, 145). Los procedimientos varían en cierto grado, pero, a grandes rasgos, se inicia con una solicitud avalada por un porcentaje del electorado (entre el 10 y el 30\%). Verificadas las firmas, la autoridad a cargo convoca a un referéndum en el cual la ciudadanía vota por sí o no a la pregunta sobre la destitución del mandatario. Cumplidos los requisitos de participación y cantidad de votos necesarios, procede la revocación y sustitución inmediata del gobernante por un suplente o vía elecciones (EBERHARDT, 2019, s.p.).

Entendemos por accountability al ejercicio del control (por vías institucionales o no institucionales) sobre los poderes públicos establecidos. Consiste en una rendición obligatoria de cuentas por parte de los gobernantes (SCHEDLER, 1999, s.p.). Si es solicitada por los otros poderes institucionales (Ejecutivo, Legislativo, Judicial y/o federales), se trata de una modalidad de accountability horizontal (como el juicio político). Si, en cambio, es requerida por los ciudadanos (como la revocatoria de mandato), estamos frente a formas de accountability vertical.

Finalmente, cuando hablamos del "hiperpresidencialismo latinoamericano", a diferencia del modelo de los EEUU (SERRAFERO, 1998, s.p.), nos referimos a la "aplicación deformada del régimen presidencial clásico, por debilitamiento de los poderes del Parlamento e hipertrofia de los poderes del presidente" (DUVERGER, 1970, 213). Es también llamado "régimen de preponderancia presidencial" (Lambert, 1974, 387), "que imita al de los Estados Unidos, pero con la diferencia de que el presidente cuenta aún con mayores poderes que en el modelo original" (SERRAFERO, 1998:166).

\section{INCORPORACIÓN DE LA REVOCATORIA DE MANDATO EN LAS CONSTITUCIONES LATINOAMERICANAS}

Uno de los mecanismos participativos incorporados en la ola de reformas constitucionales iniciada a fines de los años 80 en América Latina fue la revocatoria de mandato. Como se dijo anteriormente, este instituto de democracia directa habilita a la ciudadanía (a partir de la presentación de un cierto número de firmas) a solicitar la apertura de un referéndum en el cual pueda decidir con su voto acerca de la continuidad o no de un representante electo. En América Latina, fue adoptada por las Constituciones nacionales de Ecuador (1998), Venezuela (1999), Bolivia (2009), Colombia (1991), Perú (1993) y Panamá 
$\left(2004^{3}\right)$. En los tres primeros países, puede incluso revocarse al presidente (EBERHARDT, 2019, s.p.).

El contexto de incorporación del referéndum revocatorio en las constituciones nacionales de estos países se vio signado por graves crisis políticas, sociales y/o institucionales que motivaron la reforma. El constitucionalismo de fines de siglo en la región resultó impactado por los dos hechos históricos más significativos de la época: la crisis política y de derechos humanos derivada del paso de dictaduras y gobiernos autoritarios (años 70) y la crisis económica relacionada con la aplicación de los programas de ajuste estructural característicos de la década de 1990 (GARGARELLA, 2014, p. 269). Las nuevas constituciones surgidas en ese período fortalecieron los compromisos cívicos previos pero mantuvieron la tradicional estructura de poder vertical.

En efecto, la adopción de programas de ajuste estructural iniciada hacia fines de los años 80, a saber: "políticas monetaristas, antiestatistas, de drástica reducción del gasto público y, sobre todo, de eliminación de los programas de protección social" (GARGARELLA, 2014, p. 275), impactó sobre el constitucionalismo de varios de los países aquí analizados. Ello, debido, por un lado, a que su llegada requirió cambios legales y en las constituciones para facilitar la aplicación de las reformas, y, por el otro, a que la crisis social generada como resultado tuvo consecuencias a nivel de las Leyes Fundamentales, en función de los reclamos por el restablecimiento de una autoridad presidencial fuerte y en cuanto al nacimiento de un constitucionalismo de carácter popular y social (Ídem).

\section{a. VENEZUELA}

La primera Constitución venezolana en incorporar la revocatoria de mandato fue la de 1999. Incluida a propuesta del entrante presidente Hugo Chávez, constituyó un eje central de su primera campaña electoral (de 1998) para el Ejecutivo nacional. En dicha campaña se planteó

3Si bien en Panamá apareció por primera vez en la Constitución de 1972, sus requisitos eran tan inalcanzablemente altos que no cuenta a ciencia cierta como un mecanismo utilizable por la ciudadanía. Por ejemplo, la solicitud debía ser firmada por el $75 \%$ del padrón. Tamaño piso sobrepasa varias veces el rango de firmas exigido por las demás constituciones (entre el 10 y el 30\% del electorado). Esta modalidad fue eliminada de la Constitución de 1983, que, en cambio, incorporó la revocatoria partidaria. Recién en 2004 se adopta una forma viable de revocatoria como mecanismo de participación y control ciudadano. 
la necesidad de convocar a una Asamblea Constituyente que incorporara a la revocatoria de mandato (entre otros cambios de relevancia) en la nueva Ley Fundamental.

La iniciativa de adopción de este mecanismo participativo (junto con varios otros) tenía un antecedente fallido en las deliberaciones de la Comisión Bicameral de Revisión de la Constitución de 1961 (la de más larga duración en la trayectoria venezolana como nación independiente), celebradas entre los años 1989 y 1992 bajo la segunda presidencia de Carlos Andrés Pérez (Acción Democrática). No obstante, este intento fracasó al ser suspendida la segunda discusión de la reforma constitucional en la Cámara de Senadores a finales de 1992 (KORNBLITH, 2014, p. 111).

Esta primera tentativa de incorporación de mecanismos de democracia directa en la Constitución (cuya reforma no se pudo concretar en esa ocasión) "formó parte de un movimiento crítico respecto del rendimiento de la democracia venezolana que, después de más de tres décadas de funcionamiento desde su instauración en 1958, mostraba signos de agotamiento y distorsiones importantes" (KORNBLITH, 2014, p. 112). En general, se criticaba la excesiva partidización de las decisiones colectivas, el monopolio de la participación y representación sociopolítica a manos de los partidos, y el manejo de la estructura social a partir de influencias de poder que favorecían el clientelismo y la corrupción.

Ante el evidente fastidio cívico manifiesto respecto de las principales instituciones de la democracia representativa, varias de las propuestas alternativas comenzaron a evidenciar un creciente interés por introducir nuevos dispositivos democráticos. La meta entonces era oxigenar el sistema político y apuntalar cambios en un modelo que se percibía como exhausto. No obstante, a esa intención inicial "la clase política fue incapaz de darle impulso decisivo, ante sus contradicciones, la ausencia de miras, y en definitiva la preocupación ante lo que se visualizaba como una suerte de caja de Pandora, que arrastraría el temor a su desplazamiento del poder" (COMBELLAS, 1999, p. 1).

El descontento colectivo no tardaría en manifestarse ante la inercia gubernamental para satisfacer sus demandas (MARTÍNEZ OLIVEROS, 2008, s.p.). Esta combinación de crisis económicas y procesos de protesta popular derivó en la destitución, a manos del Congreso, del entonces presidente Carlos Andrés Pérez en 1993, como resultado de un proceso que le fuera iniciado por corrupción (GARGARELLA, 2014, p. 277). Este presidente había impulsado cambios profundos en la política económica y social del Estado y en su aparato, los que, fundamentados en la profundización de la democracia, estaban dirigidos primordialmente a 
implantar una economía neoliberal (OCHOA ENRÍQUEZ; CHIRINOS ZÁRRAGA, 1999, s.p.).

Previo a su destitución, Pérez había sufrido dos intentos fallidos de golpe de Estado. El primero, impulsado por militares comandados por Chávez en febrero de 1992 bajo el argumento de los elevados niveles de corrupción y el deterioro de la democracia. Este intento, a diferencia de los ocurridos en los años 50 y 60, no fue rechazado por las multitudes en la calle. El segundo, fue promovido en noviembre por un movimiento nuevo liderado por Gruber Odreman, marino militar y camarada de armas de Chávez. Tales tentativas favorecieron el conocimiento de Chávez como líder carismático y abrieron el debate a favor de una Asamblea Constituyente propuesta por los insurrectos.

Tras el fracaso en términos sociales de la gestión del siguiente presidente electo, Caldera, buena parte del colectivo vio en Chávez a un líder, en un momento en el que los liderazgos y los partidos perdían toda credibilidad. Fue en rechazo a esa política tradicional de partidos y en representación de un nuevo movimiento con carácter nacionalista, que Hugo Chávez se postuló como candidato presidencial en las elecciones de 1998. Su proyecto político innovador presentaba dos ejes fundamentales: la elección de una Asamblea Constituyente y la elaboración de un nuevo texto constitucional donde se contemplará, entre otras medidas, la revocatoria de mandato para los cargos de elección popular.

En este contexto, y en el de una baja de los precios del petróleo, Chávez triunfó con uno de los más altos porcentajes obtenidos hasta entonces en la historia democrática del país: el $62.46 \%$ de los votos (3.673.685 sufragios $)^{4}$, con una relativamente baja abstención del 36,54\% $(4.014 .298)^{5}$ (recordemos que en Venezuela el voto es no obligatorio).

El triunfo electoral de Chávez marcó una nueva etapa en la reforma del Estado venezolano, signada por un profundo cambio en la orientación política y en el aparato administrativo. Esta presidencia se erigía sobre un gran apoyo popular, adverso a los partidos que históricamente habían gobernado la democracia desde los años 50. Su discurso diferencial se expresaba abiertamente a favor de los sectores sociales más débiles, lo que asustaba a los grupos económicos nacionales e internacionales y desataba la crítica de los adversarios. Su lema era la denuncia contra la corrupción de los viejos partidos, la puja por un proceso constituyente 
destinado a profundizar la democracia, y el rechazo a la política neoliberal y al capitalismo salvaje (OCHOA ENRÍQUEZ; CHIRINOS ZÁRRAGA, 1999, p. 1).

El día de la asunción, y bajo una asamblea con mayoría opositora, Chávez juró sobre la "moribunda" Constitución de 1961. A poco de haber asumido su primer mandato, el 4 de febrero de 1999, convocó por decreto a un referendo consultivo sobre la posibilidad de celebrar una Asamblea Nacional Constituyente (ANC), como medio para refundar la República.

La Constitución entonces vigente no contemplaba la posibilidad de llamar a ANC. Solo permitía el procedimiento de enmienda (art. $245^{\circ}$ ) y la reforma constitucional (art. $246^{\circ}$ ). Tampoco incluía al mecanismo utilizado en esa oportunidad para su convocatoria: el referendo consultivo nacional vinculante. Este último constituía una adquisición reciente de los promotores de los mecanismos de democracia directa, plasmado en la Ley Orgánica del Sufragio y Participación Política (LOSPP), del 10 de diciembre de 1997.

Por ese motivo, el llamado a referendo consultivo desató una amplia contienda política y jurídica. Finalmente, la Corte Suprema de Justicia sentenció a favor de Chávez, despejando el camino para consultar al pueblo sobre la convocatoria a una ANC. Formalizada la convocatoria por Decreto, se desató nuevamente la crítica y se exacerbó el conflicto político. Una iniciativa que a ojos del primer mandatario y de su grupo de apoyo pondría fin a la crisis institucional venezolana, era en cambio percibida por la oposición como una amenaza para la libertad del sistema político (Martínez Oliveros, 2008, s.p.).

La consulta se celebró el 25 de abril de 1999, con una muy elevada abstención: el $62,35 \%$ de los 6.850 .747 empadronados. Las preguntas realizadas fueron dos: por un lado, si se convocaba a una ANC para transformar el Estado y crear un nuevo ordenamiento jurídico que permitiera el funcionamiento efectivo de una Democracia Social y Participativa. Esta pregunta logró un 87,75\% de respuestas positivas (3.630.666 votos) y un 7,26\% de negativas (300.233 votos). Por otro lado, si se estaba de acuerdo con las bases propuestas por el Ejecutivo Nacional para la convocatoria a la ANC, examinadas y modificadas por el CNE. El sí obtuvo el $81,74 \%$ de los votos (3.382.075) y el no, el $12,75 \%(527.632)^{6}$.

A pesar de la baja participación en la consulta (el 37,65\%), el oficialismo interpretó los resultados como un firme respaldo popular a sus propuestas. Ese triunfo, dio pie a la celebración

6 Resultados Electorales Referendo Consultivo Nacional 25/04/1999, Consejo Nacional Electoral, Venezuela. 
de elecciones de convencionales constituyentes, en un contexto de conflictividad entre el presidente y la vieja elite política. De los 131 cargos que componían la ANC, tres estaban reservados para delegados indígenas (quienes asimismo ganaron otros dos de los 128 restantes en disputa). En las elecciones constituyentes del 25 de julio (que contaron con un 53,77\% de abstención), la oposición solo obtuvo cinco bancas, mientras que el "Polo Patriótico" de Chávez se alzaba con los restantes 121 cargos (a los que debían sumarse los cinco acumulados por representantes indígenas afines). La influencia chavista se extendía entonces sobre el 96,18\% de la ANC.

En adelante, se iniciaba la etapa de elaboración de una nueva Constitución; reorganización del aparato jurídico, político y administrativo; y construcción de un sistema democrático pretendidamente social y participativo. La ANC se declaró poseedora de facultades originarias y subordinó a sus dictados los poderes establecidos (COMBELLAS, 1999, p. 1).

Además (y por encima) de la incorporación de la revocatoria de mandato y de otros mecanismos participativos, las amplias facultades de la ANC habilitaron la aprobación de profundas reformas estructurales, las que incluyeron la reorganización de los órganos del Poder Público y, en especial, los referidos al Poder Legislativo y al Poder Judicial.

En cuanto a las reformas participativas o democratizadoras, se enfatizaron y jerarquizaron numerosos valores y principios como la libertad, la igualdad, la democracia, la responsabilidad social, el pluralismo político $\left(\operatorname{art} .2^{\circ}\right)$. Uno de los fines atribuidos al Estado fue el ejercicio democrático de la voluntad popular (art. $3^{\circ}$ ). Se reconoció que la soberanía residía intransferiblemente en el pueblo, quien la ejercía directa o indirectamente (esto último mediante la elección de los representantes del Poder Público) (art. $5^{\circ}$ ). La forma de gobierno de la República Bolivariana sería democrática, participativa, electiva, pluralista y de mandatos revocables $\left(\right.$ art. $6^{\circ}$ ). Nótese aquí la primera mención al instituto revocatorio.

Algo más adelante, en el Capítulo IV, se establecieron los Derechos Políticos y del Referendo Popular. Aquí se ensalzó la participación del pueblo en la formación, ejecución y control de la gestión pública: el Estado y la sociedad debían facilitar la generación de las condiciones más favorables para su práctica (art. $62^{\circ}$ ). Los artículos $71^{\circ}$ a $74^{\circ}$ regulaban el mecanismo del referendo. Entre sus modalidades se incluyeron las consultas convocadas por el Ejecutivo, por el Legislativo o por un porcentaje de la ciudadanía (10\% del padrón). Otra forma de referendo fue la aquí analizada revocatoria de mandato, que alcanzaba a todos los cargos de elección popular (art. $72^{\circ}$ ) incluyendo nada menos que al del presidente. A su vez, el artículo 
$204^{\circ}$ inc. 7 otorgó poder de iniciativa de ley a los electores en un número no menor al cero coma uno por ciento de los inscritos en el padrón. Otra de las innovaciones en términos participativos fue la previsión de que toda revisión constitucional debía someterse a referendo popular (art. $341^{\circ}$ inc. 3), ello en virtud de la jerarquía de las normas constitucionales y del valor de la democracia participativa.

Al menos en términos discursivos, la lectura es que el principio de democracia participativa fue adoptado por los partidarios del presidente en medio de un turbulento período de cambio, a fin de incluir la participación directa del pueblo en la toma de decisiones. Sin embargo, a juicio de Fraschini, el fin último era "esquilar el poder de las elites políticopartidistas" $(2013,81)$.

En efecto, esta inclinación participativa puede interpretarse como una dádiva populista dirigida a compensar (o a disimular) el refuerzo de las prerrogativas presidenciales (parte orgánica) plasmado en: la reelección inmediata y por única vez del presidente (art. $230^{\circ}$ ); la extensión de su mandato a seis años (art. $230^{\circ}$ ); la elección presidencial por mayoría simple a una vuelta (desechando el balotaje) (art. 227 ); la potestad de nombrar y remover al vicepresidente (art. $236^{\circ}$ inc. 3); el poder de dictar decretos con fuerza de ley (art. $236^{\circ}$ inc. 8) y de reglamentar leyes (art. $236^{\circ}$ inc. 10); la posibilidad de declarar estados de excepción y decretar la restricción de garantías en los casos previstos en la Constitución (art. 236 inc. 7); la facultad de promover ascensos militares prescindiendo de la autorización del Legislativo (art. $236^{\circ}$ inc. 6); la flexibilización (a favor del Ejecutivo y en detrimento del Legislativo) de la facultad de fijar el número, la organización y la competencia de los ministerios y otros organismos de la Administración Pública Nacional (art. $236^{\circ}$ inc. 20); y, sobre todo, la atribución presidencial de disolver a la Asamblea Nacional (art. $236^{\circ}$ inc. 21). Como señala Fraschini, tales recursos institucionales conquistados por el primer mandatario tras la reforma serían vitales para la transformación social que desplegaría en los años siguientes $(2013,79)$.

En la referencia que sigue, se sintetiza la doble cara de la reforma:

Para algunos autores, estas modificaciones constitucionales, en especial las que amplían la participación ciudadana en la arena electoral, eran positivas, ya que permitían extender los marcos de la democracia representativa (Pérez Jiménez, 2003), en tanto que para otros se trataba de un texto constitucional que fortalecía la figura del primer mandatario acrecentando el poder presidencial y debilitando el legislativo (MÁRQUEZ, 2004; FRASCHNI, 2013, p. 81). 
Esta nueva Ley Fundamental, la número 26 de la historia constitucional venezolana, fue aprobada por la ANC el 19 de noviembre de 1999. El 15 de diciembre, y antes de ser publicada, fue sometida a referéndum, obteniendo el apoyo de un 71,78\% del total de votos válidos emitidos (y la negativa del 28,22\%). La abstención electoral ascendió al 54\% del padrón. En realidad, la nueva Constitución fue aprobada por un 33\% de los electores, habiendo votado solo el $46 \%$ de los inscriptos (COMBELLAS, 2002, 10) ${ }^{7}$.

Entre los meses de abril y diciembre (de 1999) se celebraron en forma consecutiva tres elecciones (dos plebiscitos y una de convencionales) que dieron por resultado un nuevo texto constitucional legitimado por la ciudadanía. La nueva Constitución introdujo cambios sustantivos en lo referido a los poderes presidenciales, incrementando los recursos de poder institucionales del primer mandatario, en especial en la relación con el Legislativo (posibilidades de decretos de acuerdo a las distintas mayorías), su influencia en los ascensos en el ámbito castrense y las facultades excepcionales con que gozará en este nuevo escenario institucional. La aprobación de este tex to constitucional permitió un nuevo llamado a elecciones que será la génesis de una nueva configuración institucional y política favorable al presidente, y traerá nuevos recursos de poder en la arena parlamentaria y regional (FRASCHINI, 2013, $82)$.

\section{b. BOLIVIA}

En este país, las primeras ideas esgrimidas en torno de la institucionalización de la revocatoria de mandato se expresaron en el año 2006, con motivo de la postulación de los candidatos del Movimiento Al Socialismo (MAS) -el partido del presidente recién asumido, Evo Morales-, a la elección de miembros de la Asamblea Nacional Constituyente (ANC), cuyo poder soberano se abocaría a refundar Bolivia (VERDUGO SILVA, 2014, p. 138).

Al poco tiempo, y a fin de que fuese debatido en la ANC, el mismo partido formuló el documento "Refundar Bolivia, Para vivir bien, Propuesta para la Asamblea Constituyente Movimiento Al Socialismo, Instrumento Político por la Soberanía de los Pueblos MAS-IPSP”. Dicho documento postulaba que "la soberanía reside en el pueblo" y que "será ejercida

7 No obstante cabe recordar que siendo el voto no obligatorio en todas sus formas en ese país, el abstencionismo electoral es previsiblemente mayor en esos casos que en los países donde el sufragio es obligatorio. 
directamente mediante los mecanismos de democracia participativa como la facultad de revocatoria de los gobernantes" (VERDUGO SILVA, 2014, p. 138).

En enero de 2007, a un año de iniciado su primer mandato, el presidente prometió enviar al Parlamento un proyecto de ley que interpretara el artículo $4^{\circ}$ de la Constitución entonces vigente (la de 2004) "para someter a referéndum revocatorio a aquellas autoridades que no cumplen en su gestión" 8 . La motivación real del primer mandatario era librarse de los más fervientes opositores a su gobierno, seis $^{9}$ de los nueve prefectos, quienes además impulsaban la autonomía departamental. Ese enfrentamiento había desatado violentos choques entre bandos opuestos y masivas movilizaciones ${ }^{10}$.

En septiembre de 2007 el presidente Morales redobló la apuesta: ahora pretendía incluir la revocatoria en la nueva Constitución. La justificación era evitar más conflictos sociales, ya que, según decía, a nivel nacional el 70\% de los conflictos provenían de las prefecturas y las alcaldías y se desataban por intereses políticos y no por reivindicaciones legítimas de la población. A su juicio, incorporar la revocatoria evitaría la proliferación de gobernantes “mandamás” o “todopoderosos” (VERDUGO SILVA, 2014, p. 138).

Tras las elecciones de convencionales constituyentes celebradas el 2 de julio, el MAS logró la mayoría en la ANC: 137 de 255 miembros. Sin embargo, no consiguió cubrir los 2/3 requeridos por la ley de convocatoria para aprobar la nueva Constitución. El fraccionamiento de la Asamblea hacía difícil la negociación y el acuerdo (DEHEZA, 2007, s.p.).

El desempeño de la ANC resultó caótico y el tratamiento de la revocatoria de autoridades fue objeto de serias disputas políticas. El 24 de noviembre de 2007, luego de más de tres meses de disturbios, la Asamblea sesionó a puertas cerradas en Sucre, en un liceo militar y sin la presencia de los asambleístas opositores al presidente. Se abría así una grave crisis política en torno de su legitimidad (VERDUGO SILVA, 2014, p. 140-141). El resultado de la jornada fue la aprobación en general de la nueva Constitución, lo que desató nuevos y violentos disturbios por parte de los contrarios a Morales.

8 "Morales propone proyecto de ley para revocar mandato de autoridades", El Universal, Caracas, $13 / 01 / 2007$.

9 Los departamentos orientales de Santa Cruz, Beni, Tarija, Pando, y los occidentales de La Paz y Cochabamba. Ver "El pulso entre el Gobierno boliviano y las regiones amenaza con extender la violencia", El País, 12/01/2007.

10 “Morales propone proyecto de ley para revocar mandato de autoridades", El Universal, Caracas, $13 / 01 / 2007$. 
Esa jugada, tachada de inconstitucional por haberse votado sin mayoría calificada, profundizó la fractura del país e impulsó los procesos autonomistas de los departamentos de Santa Cruz, Pando, Beni, Tarija, Cochabamba y ahora también Chuquisaca (departamento occidental gobernado por el MAS), en su pretensión de obtener poderes federales. El 9 de diciembre la ANC, trasladada a Oruro, aprobó en detalle la nueva Constitución sin la presencia de la oposición derechista. Sin embargo, esta quedaría en un limbo hasta enero de 2009, momento en que, tras dos intentos fallidos (mayo y diciembre de 2008) fue ratificada en referendo ${ }^{11}$.

En lo que hace a la participación ciudadana y a la ampliación democrática, esta nueva Ley Fundamental incorporó como novedad el reconocimiento a la libre determinación de los pueblos originarios (art. 2). El artículo $7^{\circ}$ determina que la soberanía, inalienable e imprescriptible, reside en el pueblo boliviano, y que se ejerce de forma directa y delegada (a los órganos del poder público). Entre los valores del Estado destaca la equidad social y de género en la participación (art. $8^{\circ}$ inc. II). La forma de gobierno adoptada es democrática participativa, representativa (por elección de los representantes) y comunitaria (por normas propias de los pueblos originarios). La democracia directa y participativa se ejerce por medio del referendo, la iniciativa legislativa ciudadana, la revocatoria de mandato, la asamblea, el cabildo y la consulta previa $\left(\operatorname{art} .11^{\circ}\right.$ ). Respecto de la revocatoria de mandato, esta es regulada por el artículo $240^{\circ}$. La misma atañe a todos los cargos, incluyendo al del presidente. Finalmente, el Título VI habilita la participación y el control social, ejercido por el pueblo soberano por medio de la sociedad civil organizada.

Sobre fines de diciembre, y ante la virulencia del conflicto, el presidente Morales no esperó a la entrada en vigencia de la nueva Constitución y entregó a su vice (máxima autoridad del Congreso Nacional), el Proyecto de Ley para convocar a referéndum revocatorio del Ejecutivo nacional (presidente y vice) y de todos los gobernadores departamentales. Con ese acto, Morales impulsaba un mecanismo ajeno a la aún vigente Constitución de 2004. El resultado fue la permanencia del presidente y su vice y de seis prefectos: los dos oficialistas de los departamentos de Oruro y Potosí y los cuatro opositores de Tarija, Santa Cruz, Pando y Beni

11 “Bolivia: Evo cosechó otra derrota, esta vez nacida de su propio riñón”, La Capital, Rosario, 30/06/2008. 
mantuvieron sus puestos. Contrariamente, los prefectos opositores de La Paz y Cochabamba fueron revocados.

En este caso, es claro que la revocatoria de mandato fue una herramienta de poder impulsada por el presidente Evo Morales para desactivar, vía la destitución de los respectivos prefectos, la oposición autonomista de los departamentos orientales ${ }^{12}$, así como para aplacar los graves disturbios ocasionados a consecuencia de dicha rivalidad. Respecto de la adopción de este mecanismo, aplica lo que Gamboa Rocabado sostiene respecto de las otras medidas democratizadoras incluidas en la Constitución: "el discurso de participación popular directa y ancestral de los pueblos indígenas fue explotado hábilmente por el MAS, que negó, simultáneamente, las demandas autonómicas de la Media Luna (la mitad del territorio, localizado en el oriente del país). Esta contradicción también puede terminar en el surgimiento de amenazas autoritarias" (GAMBOA ROCABADO, 2010, p. 164).

En efecto, la revocatoria de mandato tuvo un lugar prioritario en la agenda presidencial desde mediados de 2006, en ocasión de la elección de los convencionales constituyentes. Prevista primero como ley basada en la interpretación del artículo $4^{\circ}$ de la antigua Constitución; luego, incorporada al articulado de la nueva Ley Fundamental y; finalmente (y ante la demora de su entrada en vigencia); enviada como proyecto de ley al Congreso, destinado a someter a revocatorio los mandatos de las máximas autoridades nacionales y federales vigentes en ese momento: presidente, vice y los nueve prefectos.

El apuro del presidente por institucionalizar este mecanismo parece deberse más a su voluntad estratégica de emplearlo como un medio para debilitar y, eventualmente, deshacerse de la oposición autonomista, que a un compromiso verdadero con la participación y el control popular. Tan es así, que Evo Morales no pudo esperar a la entrada en vigencia de la nueva Constitución para que la ciudadanía decidiera por sí misma si quería iniciar algún proceso en contra de algún prefecto. Por el contrario, la aplicó a través de una ley ad hoc, creada en exclusiva para implementar el mecanismo en esa coyuntura.

De hecho, la Ley N 3.850 de Referéndum Revocatorio del Mandato Popular (LRRMP), del 12 de mayo de 2008, contenía los nombres de los mandatarios expuestos a revocatoria. Asimismo, prescindió de la recolección de firmas, siendo este el primer paso obligatorio para 
la implementación del mecanismo por parte de la población. Dicho acortamiento del proceso tuvo que ver con que el mismo no fue impulsado por la ciudadanía (en virtud del descontento con algún gobernante) sino por el propio presidente (para afirmarse en el poder y debilitar o eliminar a la oposición). De este modo, y lejos de su espíritu democrático y participativo, la revocatoria se convertía en una herramienta de uso estratégico en la competencia política a manos del presidente.

Nótese que tanto la reforma de Venezuela (1999) como la de Bolivia (200) fueron impulsadas por los presidentes a cargo, y ambas se propusieron como refundaciones radicales del Estado. En los dos casos, la incorporación de la revocatoria de mandato fue producto de la iniciativa de los primeros mandatarios en un intento (más directo en el segundo caso) de fortalecer el propio poder y debilitar el de los otros órganos de gobierno (Legislativo, Judicial y federal). Si en la Constitución de Chávez el revocatorio fue un plato urdido fuerte para compensar o disimular la sobrecarga de atribuciones en el presidente y el debilitamiento de los otros poderes, en manos de Morales este mecanismo actuó como un disuasivo de la oposición autonomista. En ambas ocasiones se lesionó la posibilidad de ejercer un efectivo control horizontal al presidente por parte de los otros poderes y se reforzaron los ya importantes atributos del ejecutivo unipersonal, poniendo en riesgo la propia vigencia de los derechos y libertades, el respeto de la pluralidad y el ejercicio mismo de la participación y el control ciudadano. Las palabras de Gamboa Rocabado aplican a ambos escenarios: "toda la hostilidad del MAS hacia los proyectos políticos de la Media Luna no es admisible ni congruente con la consolidación de un régimen democrático" $(2010,164)$.

\section{c. ECUADOR}

En este país, la incorporación de la revocatoria de mandato (junto con la de otras herramientas de democracia directa), data de la Asamblea Constituyente de 1997, en medio de un clima en el cual varios grupos de la sociedad civil comenzaban a mostrar un mayor interés por participar e intervenir directamente en la toma de decisiones públicas, así como por controlar el accionar de sus representantes. La expansión de las instituciones de participación ciudadana por aquel entonces respondió a la necesidad de complementar el desempeño de la democracia representativa (CASTELLANOS, 2014, p. 84).

El escenario de fondo era el de una abierta disputa entre los poderes de gobierno en torno de la ineficiencia y baja calidad democrática de las instituciones, junto con la pérdida de legitimidad y desconfianza hacia los partidos tradicionales. Todo lo cual "incrementó la presión 
por reformas estructurales e institucionales capaces de garantizar la soberanía popular y evitar la conflictividad interna" (CASTELLANOS, 2014, p. 84).

En ese mismo año 1997 había tenido lugar la escandalosa remoción del presidente Abdalá Bucaram. El Congreso Nacional lo declaró incompetente por incapacidad o trastorno mental. Los motivos fueron: su política monetaria, las subas de precio, la corrupción de sus funcionarios, escándalos personales y nepotismo (CASTELLANOS, 2014, p. 85). Si bien el procedimiento tuvo vicios de legitimidad y falta de garantías constitucionales, contó con el respaldo de gran parte de la ciudadanía, sindicatos y Organizaciones No Gubernamentales. La vicepresidenta, Rosalía Arteaga, solo asumió por 24 horas ya que el Congreso designó en su lugar a su propio presidente, Fabián Alcón Rivera.

El presidente interino llamó a consulta popular, celebrada el 25 de mayo, donde, entre otras varias cuestiones, se convalidó la destitución y el reemplazo presidencial, se resolvió el establecimiento de la Asamblea Constitucional con poderes plenipotenciarios para redactar una nueva Ley Fundamental, y se acordó la adopción constitucional del principio de revocatoria para los mandatarios elegidos que incumplieran normas morales, legales y de eficiencia en el ejercicio de sus funciones.

La pregunta por la revocatoria fue la número 13, la última antes de la disposición final. La participación ascendió al 59\% del padrón. Por el SI, se acumularon 1.615.292 votos $(39,64 \%)$. Por el NO, 1.065 .850 (26,15\%). Hubo 460.098 (11,29\%) votos en blanco y 933.379 $(22,9 \%)$ nulos. El total de votos positivos fue de 2.681.142. De ese total, el porcentaje de población a favor de introducir la revocatoria en la letra constitucional fue del 60,24\% mientras que el 39,76\% prefirió excluirla.

A pesar del apoyo popular recolectado en el referéndum, "los miembros de la asamblea constituyente de 1998 no se orientaron totalmente hacia este precepto, ya que este mecanismo no estaba diseñado para permitir su ejercicio autónomo" (CASTELLANOS, 2014, p. 86). La nueva Constitución de 1998 cumpliría entonces con la introducción de la revocatoria de mandato, pero, debido a las numerosas restricciones contenidas en su previsiones legales (que dificultaban su activación en los subniveles de gobierno y no permitían la remoción del presidente), quedaría inactiva por más de una década (CASTELLANOS, 2014, p. 84).

Esta Constitución, al igual que la de Venezuela, se incluyen entre aquellas cartas políticas que, "por un lado, buscan maximizar los derechos y proponen mecanismos generosos de participación popular, mientras mantienen, al mismo tiempo, organizaciones políticas fuertemente centralizadas y verticalizadas" (GARGARELLA, 2014, p. 310). 
Los avances constitucionales que presenta la Constitución de 1998, y que tiene que ver con la importancia de su parte declarativa o dogmática se corresponden con un agudo retraso y disfuncionalidad de su parte orgánica, esto es, de aquel conjunto de estructuras institucionales por medio de las cuales las políticas públicas podrían en efecto funcionar como respuesta a esa estructura de derechos (GARGARELLA, 2014, p. 315).

Posteriormente, la acción directa de los ecuatorianos, canalizada de modo informal, tendría poder de presión sobre la destitución de otros dos presidentes: Jamil Mahuad Witt (2000) y Lucio Gutiérrez (2005). En el marco “de la pérdida de legitimidad de las instituciones representativas, ambos procesos acentuaron la crisis de confianza en las acciones gubernamentales" (CASTELLANOS, 2014, p. 86).

La visible pugna de poderes entre los miembros del Congreso y el presidente durante sucesivos gobiernos ha sido la variable constante que ha influido al conflicto e inestabilidad del sistema político en el país. Estos hechos han provocado la intervención de la sociedad civil. La "rebelión de los forajidos"13, en 2005, demostró el descontento con el sistema político al constatar que las cortes de justicia eran poco fiables, y el proceso administrativo estaba plagado de corrupción y clientelismo (CASTELLANOS, 2014, p. 87).

Estos acontecimientos fueron considerados como procesos de democracia directa desinstitucionalizada, ocurridos en ausencia de un mecanismo institucional que permitiera viabilizar la remoción de la autoridad evitando enfrentamientos internos que desestabilicen el sistema representativo. Siendo la destitución de 2005 el agravante para considerar la reformulación jurídica de la revocatoria (CASTELLANOS, 2014, p. 87).

Las sucesivas crisis y protestas de la década de dos mil condujeron a una revisión del diseño constitucional en 2008 (CASTELLANOS, 2014, p. 91). Tal escenario, prepararía el terreno propicio para la extensión y flexibilización de los mecanismos participativos y de control. En efecto, "la ausencia o disfuncionalidad de intermediarios como los partidos políticos amplió la brecha entre los ciudadanos y el gobierno, y motivó la apertura de canales de participación política accesibles desde la ciudadanía” (CASTELLANOS, 2014, p. 86-87).

13 La Rebelión de los Forajidos fue un movimiento golpista civil, posteriormente respaldado por el comando conjunto de las fuerzas militares, que tuvo lugar del 13 de abril al 20 de abril de 2005 en Quito, y que derrocó el gobierno de Lucio Gutiérrez sustituyéndolo por Alfredo Palacio, el vicepresidente. 
En realidad, la idea redactar una nueva Constitución para el Ecuador formó parte de la campaña presidencial de Rafael Correa, con vistas a las elecciones de 2006 en las que resultó vencedor. Tras intensas luchas con el Congreso Nacional (de mayoría opositora) logró convocar a una consulta popular sobre su propuesta de formar una Asamblea Constituyente. El 81,72\% del electorado votó a favor. En las elecciones de asambleístas del 30 de septiembre de 2007, el oficialismo obtuvo 80 de las 130 bancas, lo que le dio la mayoría.

En lo que hace a las reformas democratizantes, el Título IV de la nueva Constitución se orientó por entero a la Participación y Organización del Poder. El mismo incluyó: los principios de la participación ( $\operatorname{art.} 95^{\circ}$ ), espacios de participación en los distintos niveles de gobierno (art. $100^{\circ}$ ), la "silla vacía" (art. $101^{\circ}$ ) para que un representante ciudadano participe de las sesiones de los gobiernos autónomos descentralizados, la iniciativa popular (art. 103º), la consulta popular (art. $104^{\circ}$ ), la posibilidad de participar de las comisiones legislativas (art. $137^{\circ}$ ) y la revocatoria de mandato (art. $105^{\circ}$ ), la que fue extendida hasta alcanzar todos los cargos de elección popular, superando en mucho la versión de 1998 en la que solo se afectaba a alcaldes, prefectos y diputados.

En cuanto a la parte orgánica, la reforma de 2008 no redujo el excesivo poder presidencial de la Constitución de 1998. A juicio de varios especialistas, este fue aumentado, al tiempo que se disminuyeron las facultades legislativas y la real participación de la sociedad. Al igual que en Venezuela, la ampliación de la parte dogmática (incluyendo la extensión del alcance de la revocatoria) vino a distraer la atención respecto del refuerzo de las potestades del presidente (parte orgánica).

De hecho, el Ejecutivo vio multiplicadas sus funciones (incluso algunas quedaron como prerrogativas exclusivas). Entre sus facultades se destacan: la exclusividad en la presentación de proyectos que creen, modifiquen o supriman impuestos, aumenten el gasto o modifiquen la división político administrativa del país $\left(135^{\circ}\right)$; la objeción total o parcial de un proyecto de ley $\left(\right.$ art. $138^{\circ}$ ); la posibilidad de enviar proyectos de urgencia en materia económica (art. $139^{\circ}$ ); la planificación del desarrollo (art. $147^{\circ}$ inc. 4); atribuciones judiciales como dar indultos por delitos comunes (art. $147^{\circ}$ inc. 8); competencias en definición de política cambiaria, crediticia y monetaria en detrimento del Banco Central (art. 261 ${ }^{\circ}$ inc. 5); y nuevas facultades legislativas como: emitir reglamentos de ejecución (art. $147^{\circ}$ inc. 13); iniciativa de ley (art. $147^{\circ}$ inc. 11); reforma constitucional (art. $442^{\circ}$ ); frente a la omisión legislativa, consagrar un proyecto de urgencia económica como decreto-ley (art. $140^{\circ}$ ); y objetar normas aprobadas por la Asamblea Nacional, por razones de oportunidad o por vicios de inconstitucionalidad ( $\operatorname{art} 138^{\circ}$ y $\left.139^{\circ}\right)$. 
Ni siquiera los nuevos controles e instituciones participativas incorporadas (la "muerte cruzada" ${ }^{14}$, las novedosas herramientas de participación -como la revocatoria y la silla vacía-, la coordinación de todos los mecanismos de control) lograron compensar el desequilibrio de poder en manos del presidente (GARGARELLA, 2014, p. 311). Ello debido a que tales instrumentos participativos y de control adquirieron luego requisitos tan elevados y difíciles de cumplir, en medio de una maraña de procedimientos burocráticos, que se volvieron inaplicables. Asimismo, el propio presidente limitó, en cuanto estuvo a su alcance, las iniciativas destinadas a poner en funciones los institutos participativos que habían sido creados en la Constituyente y por él apoyados (312-313).

Como señala Gargarella, “el devenir de la práctica constitucional mostró los límites de algunas de las expectativas creadas por la letra de la Constitución” (2014, p. 312): ya que el hiperpresidencialismo reforzado y centralizado terminó por bloquear la puesta en marcha de muchas de las iniciativas participativas incorporadas, y porque la misma dinámica del régimen derivó en aumentar los propios poderes presidenciales. Frente a una Constitución desbalanceada en favor de la parte orgánica y en detrimento de la dogmática, "no había razones para sorprenderse frente a una performance pálida o deslucida por parte de las nuevas cláusulas participativas" (Ídem). Al igual que en Venezuela, en este país, la ampliación de los derechos de participación y control ciudadano (como la revocatoria) fue apenas un maquillaje para disimular la ampliación de poderes en el Ejecutivo.

\section{d. PERÚ}

La primera Constitución nacional peruana en incorporar la revocatoria de mandato (junto con otros mecanismos de democracia directa) fue la de 1993, impulsada por el entonces presidente Alberto Fujimori tras su "autogolpe" y cierre del Congreso.

Esta novedosa incorporación fue posible gracias a la confluencia de "dos vertientes contrapuestas: la izquierda y el fujimorismo" (TUESTA SOLDEVILLA, 2014, p. 9). La izquierda tenía sendos antecedentes de crítica a la formalidad e insuficiencia de la democracia representativa. Tras el gobierno militar que dirigió el país de 1968 a 1980, la llegada al poder

14Además del juicio político al presidente (art. $129^{\circ}$ ) la Asamblea Nacional podrá destituirlo por arrogarse funciones que no le competan o por grave crisis política y conmoción interna (art. $130^{\circ}$ ). Ahora bien, como el presidente tiene la misma facultad respecto de la Asamblea (art. $148^{\circ}$ ) se la ha llamado "muerte cruzada". 
de la Izquierda Unida en varios municipios alentaría el desarrollo de la noción participativa de la democracia en estos ámbitos, donde la ciudadanía jugaría un papel de mayor trascendencia y más activo en la gestión local. Por su parte, el gobierno fujimorista también alentó la organización y participación ciudadana, pero desde una mirada de profunda crítica a los partidos y a los poderes instituidos, discurso que prepararía el terreno para el autogolpe de 1992 (Ídem).

Ciertamente, Alberto Fujimori ejerció la presidencia entre 1990 y 2000, con la agrupación de centro derecha por él fundada en 1989: "Cambio 90". Vencido el mandato del centroizquierdista Alan García Pérez; cuya presidencia se había caracterizado por una grave crisis económica hiperinflacionaria, el incremento de la violencia terrorista bajo Sendero Luminoso y el descrédito de las fuerzas políticas acusadas de corrupción; Fujimori impulsó desde el gobierno un duro plan de ajuste económico para paliar la adversa situación.

Desde sus comienzos, lanzó una premeditada campaña de desprestigio contra el Poder Judicial y, más aún, contra el Congreso, al cual acusó de generar ingobernabilidad y entorpecer las medidas necesarias para organizar el Estado ${ }^{15}$. El 5 de abril de 1992, con el apoyo del ejército, encabezó un autogolpe que cerró el Legislativo, abolió la Constitución de 1979, e intervino el Palacio de Justicia. Comenzaba así el llamado "Gobierno de Emergencia y Reconstrucción Nacional", alentando cierta expectativa en la gente que, ante la creciente amenaza de los grupos terroristas, apoyó en su mayoría la nueva situación.

Según Rospigliosi, la intención oculta del presidente era la de "gobernar con poderes absolutos, sin parlamento y recurriendo a plebiscitos cada cierto tiempo" $(1998,408)$. No obstante, "la condena que recibió de parte de la comunidad internacional, lo obligó a modificar parcialmente su esquema y a convocar a elecciones para un Congreso Constituyente" (Ídem). Fue "una solución de compromiso para tratar de superar la crisis originada por el golpe" (Ídem). Estas se celebraron el 22 de noviembre de 1992, con el fin de conformar un "Congreso Constituyente Democrático" encargado de dictar una nueva Constitución para reemplazar la de 1979.

Al parecer, Fujimori no tenía en sus planes convocar a un Congreso Constituyente, ni tampoco gobernar con un Parlamento (aunque tuviera mayoría propia) en el que sus propuestas fueran puestas en discusión. Sin embargo, debió ceder a la presión internacional "porque todo

15 “Alberto Fujimori”, Biografías y Vidas. La enciclopedia biográfica en línea, en http://www.biografiasyvidas.com/biografia/f/fujimori.htm. 
su esquema económico (y en parte su estabilidad política) reposaba sobre las posibilidades de normalizar las relaciones con los organismos multilaterales, rotas durante el gobierno anterior" (ROSPIGLIOSI, 1998, p. 408).

Los partidos de oposición y la mayoría de los ciudadanos tampoco se mostraban muy entusiastas frente a la elección. Los primeros, porque no confiaban en la autenticidad del proceso ni en la independencia y autonomía del Congreso, y porque no tenían perspectivas de ganar una cantidad significativa de escaños. Los segundos, porque la propuesta no les resultaba especialmente atractiva, en medio de un clima de crisis de la representación, de desprestigio de los partidos y de las instituciones representativas, que se reflejó en una baja participación en las urnas (ROSPIGLIOSI, 1998, p. 409).

El Congreso estaría constituido por 80 congresistas, muy reducido en comparación con el Parlamento, de 240 miembros. Dicho tamaño reducido se debía a que Fujimori no contaba con muchas personas de su confianza para integrar una lista. Tampoco tenía un partido político fuerte y con presencia a nivel subnacional, por lo cual instituyó un distrito único. Además, un Congreso pequeño sería más fácil de manipular por el gobierno que uno de mayores dimensiones (ROSPIGLIOSI, 1998, p. 410).

El Gobierno de Emergencia y Reconstrucción Nacional convocó los comicios con la resistencia de varios de los partidos tradicionales del país, como el centro-izquierdista Partido Aprista Peruano (APRA o PAP); Acción Popular (AP); Izquierda Unida (IU); y Libertad, entre otros. Ninguna de esas agrupaciones participó de la elección debido a su disconformidad con el gobierno de Fujimori (al que consideraban ilegítimo y no querían convalidar), por la desconfianza que les despertaba el proceso en cuanto a su imparcialidad y por la conciencia de su debilidad para competir a nivel nacional contra el oficialismo. En cambio, el Partido Popular Cristiano (PPC), de centroderecha, y otras agrupaciones menores o emergentes, aceptaron participar del Congreso. El gobierno estaría representado por la alianza oficialista formada por el decadente Cambio 90 y el recién fundado para la ocasión partido Nueva Mayoría (C90-NM).

Las elecciones se celebraron en un clima de "inseguridad, incertidumbre y cambios constantes" (Rospigliosi, 1998, 410), bajo una ley electoral diseñada a conveniencia del oficialismo, sin tomar en cuenta la opinión de la oposición y con una excesiva presencia de las Fuerzas Armadas. De los 18 grupos políticos que se presentaron a la contienda solo 10 obtuvieron representación en el Congreso. A pesar de presentarse como independientes u opositores, una vez en el Congreso, muchos terminaron alineándose con el fujimorismo. 
Tras los comicios se declaró el triunfo de la alianza fujimorista Nueva Mayoría-Cambio 90, que obtuvo, con el 37,5\% de los votos emitidos, 44 de los 80 escaños en juego: la mayoría absoluta. En segundo lugar quedó el PPC (con un escaso 7,4\% de los votos válidos), que obtuvo 8 bancas. Luego siguieron varias listas electorales de nuevos movimientos. Hubo un ausentismo del $27,8 \%$ y un $23,9 \%$ de votos blancos y nulos, evidenciando una gran apatía cívica, producto de la crisis económica y representativa, así como de la desintegración social y política (ROSPIGLIOSI, 1998, p. 415-416).

Al igual que lo ocurrido con Chávez, la mayoría propia en el Congreso Constituyente permitió a Fujimori elaborar una Constitución acorde con su doble deseo: implantar el neoliberalismo económico y desbalancear la distribución de poderes a favor del presidente y en detrimento del Congreso. La incorporación de instrumentos de democracia directa y de rendición de cuentas, como la revocatoria de mandato (art. $31^{\circ}$ ), sumó créditos a su estrategia de aplacar la crisis institucional, revertir la reprobación internacional respecto de la irregularidad de su gobierno y desviar la atención de las modificaciones hiperpresidencialistas.

La cantidad de reformas realizadas respecto de la Constitución de 1979 fue relativamente baja, pero de gran impacto en el régimen político. Entre los principales cambios que hacen a los derechos participativos, al control y a la estructura de poderes, además de la adopción de la revocatoria (aunque solo sea para autoridades regionales y locales), destacamos: la incorporación del referéndum para la reforma total o parcial de la Constitución, la aprobación de leyes, ordenanzas municipales y materias relativas al proceso de descentralización (art. $32^{\circ}$ ); la reelección presidencial inmediata o consecutiva, debiendo luego dejar pasar un período para volver a presentarse (art. 112 $)$; la ampliación de las facultades del presidente para adoptar medidas extraordinarias mediante decretos de urgencia con fuerza de ley en materia económica y financiera (art. $118^{\circ}$ inc. 19) y para disolver el Congreso si éste censurase a dos Consejos de Ministros $^{16}$ (debiendo fijarse la fecha de elecciones para un nuevo Congreso -art. $134^{\circ}$ ); el reemplazo de la bicameralidad del Poder Legislativo por la unicameralidad (art. 90); la restricción de la atribución del Congreso de aprobar tratados internacionales antes de la ratificación presidencial solo respecto de aquellos que versasen sobre ciertas materias específicas (art. $56^{\circ}$ ), dejando en libertad al presidente para celebrar cualquier otro tratado sin

16 En la Constitución de 1979 el Presidente podía disolver la Cámara de Diputados luego de la censura a tres consejos de ministros (art. $\left.227^{\circ}\right)$. 
aprobación parlamentaria previa (art. 57 $)$; la eliminación de la ratificación del Senado sobre el nombramiento presidencial de los embajadores (art. $118^{\circ}$ inc. 12); la eliminación de la ratificación del Congreso de los ascensos de las Fuerzas Armadas o altos rangos militares aprobados por el presidente (art. $172^{\circ}$ ); la limitación de la atribución parlamentaria referida a la aprobación de tributos, salvo que fuese solicitada por el Ejecutivo (art. $79^{\circ}$ ); el establecimiento de la Defensoría del Pueblo (art. $161^{\circ}$ ).

A diferencia de la Constitución de 1979, resultado de un consenso multipartidario a favor de establecer una institucionalidad democrática tras largos años de dictadura, esta nueva carta política de 1993 fue producto de un autogolpe y de un Congreso constituyente compuesto por amplia mayoría oficialista, con una marcada ausencia de las fuerzas políticas tradicionales opositoras y escaso debate. El sistema de gobierno mixto heredado de la anterior Constitución terminó exagerando el rol del presidente y quebrando el equilibrio de poderes en pos de un hiperpresidencialismo. Al igual que en Venezuela, las concesiones a la democracia participativa (parte dogmática) debieron convivir con un presidencialismo exacerbado (parte orgánica).

La nueva Constitución fue aprobada en referéndum popular el 31 de octubre de 1993 con el 52,24\% de los votos. Los partidos opositores bregaron por el $\mathrm{NO}$, denunciando la ilegalidad e inconstitucionalidad de origen de dicha normativa. Si bien ganó la opción oficial, el triunfo no fue arrollador, evidenciando la poca credibilidad de todo el proceso.

\section{CONSIDERACIONES FINALES}

Desde fines de los años 80, la mayoría de las constituciones en América del Sur incorporaron y/o ampliaron sus mecanismos de democracia directa. Esta tendencia se orientó a alcanzar mayores niveles de participación y accountability vertical ciudadana, con el fin de corregir la crisis de representación y hacer frente a los problemas de gobernabilidad. Así, el proceso de democratización política fue de la mano de una proliferación de vías de participación y control cívico, tanto formales como informales.

Uno de los mecanismos incorporados, muy destacado en términos de su potencial participativo y de control hacia los gobernantes, es la revocatoria de mandato. Este instituto, permite a los ciudadanos poner fin al mandato de un representante antes de que finalice su período, ubicando a la soberanía popular por encima de los mandatarios. En caso de alcanzar al Ejecutivo nacional, la revocatoria flexibiliza el funcionamiento del presidencialismo, volviéndolo más apto para superar situaciones de graves crisis políticas asociadas a la figura del primer mandatario. 
En América Latina, el proceso de introducción de este mecanismo en las constituciones y leyes de varios de sus países mostró ciertos rasgos comunes, como graves crisis políticas, sociales y/o institucionales (Venezuela, Bolivia, Ecuador y Perú), o el viraje a gobiernos de izquierda o socialistas (Chávez, Morales, Correa); y también, algunos matices diferenciales, como la adopción de políticas neoliberales (Fujimori) o el enfrentamiento con los prefectos opositores de algunos departamentos (Morales).

No obstante, el aspecto más saliente en cuatro (Venezuela, Bolivia, Ecuador y Perú) de las seis constituciones que incorporaron la revocatoria de mandato desde los años 90 es que semejante apuesta a la participación y al control ciudadano se vio empañada o contrarrestada por una simultánea concentración y aumento de facultades en el presidente. Este desequilibrio de poderes a favor del Ejecutivo, sumado a los altos requisitos impuestos al revocatorio, tendieron a neutralizar su potencial de accountability y, en algunos casos, llegaron a convertirlo en un instrumento más de disputa política al servicio de los mandatarios.

El presente artículo se abocó a indagar el proceso de incorporación de la revocatoria de mandato en dichas constituciones, a fin de conocer el contexto de la reforma de la que fueron parte. La inquietud era comprender qué gobernantes y bajo qué circunstancias decidieron adoptar una herramienta institucional destinada a la interrupción anticipada de sus propios mandatos, decisión que, al menos, resulta paradójica o contradictoria. La pregunta subyacente es: ¿por qué un gobernante impulsaría un mecanismo creado para ser usado en su propia contra? ¿Por qué un mandatario electo adoptaría por iniciativa propia una herramienta que pone a la voluntad popular por encima de sus poderes durante la mayor parte de su mandato? ¿Por qué impondría voluntariamente tamaño factor de debilidad o vulnerabilidad sobre sí mismo?

Numerosas lecturas se han inclinado a entender las reformas constitucionales recientes en Latinoamérica como procesos que tanto fortalecieron como limitaron el presidencialismo regional. Mientras algunas cláusulas confirieron mayores capacidades al presidente, dicen, otros cambios "compensatorios" expandieron las capacidades de los ciudadanos para controlar a sus representantes (GARGARELLA, 2014, p. 288). La adopción de la revocatoria de mandato, que incluso en algunos países llegó hasta alcanzar a la figura presidencial (Venezuela, Bolivia y Ecuador), se incluye entre los segundos.

Sin embargo, tal efecto "compensatorio" no es realmente cierto (GARGARELLA, 2014, p. 288-289). Las interpretaciones que así lo sostienen asumen en forma errónea una relación igualitaria entre las diferentes esferas de la Constitución, cuando solo en una de ellas se 
encuentra el verdadero "motor" del régimen: la parte orgánica, referida a la organización del poder.

Por otro lado, dichas miradas descuidan el aspecto dinámico de la Constitución, limitándose a una lectura estática que toma más en cuenta el objetivo de la reforma (lo declamativo) que lo que resulta esperable (real) de su puesta en marcha, dada una cierta distribución de poderes. A juicio de Gargarella, "son habitualmente los poderes establecidos los que tienen 'la llave' capaz de 'activar' -o desactivar- los nuevos mecanismos participativos" (2014, 289).

Finalmente, tales lecturas tampoco toman en cuenta el contexto e historia de la región que permite reconocer la posición de privilegio en que han quedado los presidentes para ejercer su dominio sobre las demás herramientas constitucionales (GARGARELLA, 2014, p. 289).

En cambio, si bien las últimas reformas constitucionales del continente han mostrado una estructura compleja según la cual, por un lado, parecían mantener o profundizar los poderes presidenciales y, por el otro, pretendían fortalecer los compromisos constitucionales ya asumidos en derechos sociales o participación política, incluyendo nuevos controles al poder; estas reformas, una vez puestas en marcha, terminaron por reforzar (o, al menos, por no debilitar significativamente) la autoridad presidencial, algo que (como vimos respecto de la revocatoria) tendía a poner en riesgo el resto de las reformas (GARGARELLA, 2014, s.p.).

En efecto, resulta lógico pensar que "los presidentes con poderes fuertes o reforzados, racionalmente, no tienden a aceptar recortes sobre su propio poder, como los que puede sugerir una ciudadanía autonomizada y/o con mayores poderes de decisión y control" (GARGARELLA, 2014, p. 309). Es decir, que si un presidente (como Chávez o Morales) impulsa una reforma estructural del Estado, y en dicha reforma incluye un mecanismo que permite destituirlo anticipadamente del supremo cargo vía votación popular, es porque de algún modo está seguro de que tal mecanismo no podrá ser empleado eficazmente en su contra, ya sea porque su exigente diseño no invita a su utilización, o porque tiene asegurada su influencia sobre los órganos de implementación (de justicia y/o electoral), o porque planea usarlo él mismo a su favor y en contra de sus adversarios.

En síntesis, en los casos de países cuyas constituciones han sido reformadas bajo un gobierno personalista con intención de instalar un orden hiperpresidencialista (como en los cuatro casos aquí estudiados), la adopción de la revocatoria puede leerse, más que como una contradicción en sí misma, como un gesto democrático participativo destinado a desviar la atención de otras reformas, más profundas, realizadas en la dirección contraria: reforzar y 
concentrar el poder en el Ejecutivo nacional. Tales mecanismos se convierten en meros "adornos" democráticos o injertos constitucionales extraños en un entorno institucional que poco margen deja a la real participación y control ciudadanos.

\section{REFERÉNCIAS}

Altman, David. Democracia Directa en el continente americano: ¿autolegitimación gubernamental o censura ciudadana?, Política y Gobierno, México D.F., XII, 2, pp. 203-232, 2005.

BucEK, Ján y SMiTh, Brian. New approaches to local democracy: direct democracy, participation and the 'third sector', Environment and Planning C: Government and Policy, 18, 1, pp. 3-16, 2000.

Castellanos, Ana Sofía. Ecuador: la transformación de las reglas del juego y sus consecuencias (1998-2013). En: WELP, Yanina y SERDÜLT, Uwe (Comp.) La dosis hace el veneno. Análisis de la revocatoria del mandato en América Latina, Estados Unidos y Suiza. Quito, Consejo Nacional Electoral - Instituto de la Democracia, 2014.

COMBELlas, Ricardo. Constitución de 1999 y la reforma política: implicaciones para la gobernabilidad democrática. In: VII Congreso Internacional del CLAD, Lisboa, 2002.

CROnIn, Thomas. Direct Democracy. The Politics of Initiative, Referéndum and Recall. Cambridge MA: Harvard University Press, 1989.

DEHEZA, Grace. Bolivia 2006: reforma estatal y construcción del poder, Revista de Ciencia Política, Santiago, vol. especial, pp. 43-57, 2007.

DUVERGER, Maurice. Instituciones políticas y derecho constitucional. Barcelona: Ariel, 1970.

EBERHARDT, María Laura. Efecto helicóptero. ¿Cómo reemplazar malos gobernantes sin golpes de Estado? Buenos Aires: Prometeo, 2019.

Eberhardt, María Laura. Siga Participando. Dedicado a los ciudadanos de las democracias reales. Buenos Aires: Prometeo, 2013.

FRASCHINI, Mariano. Los liderazgos presidenciales de Hugo Chávez y Álvaro Uribe: ¿Dos caras de un mismo modelo de ejercicio del poder? 2013. 408. Tesis doctoral - Doctorado en Ciencia Política - Universidad Nacional de General San Martín, Buenos Aires.

GAmBOA RocABADO, Franco. Transformaciones constitucionales en Bolivia. Estado indígena y conflictos regionales, Colombia Internacional, 71, pp. 151-188, enero-junio 2010.

Gargarella, Roberto. La sala de máquinas de la Constitución. Buenos Aires: Katz Editores, 2014.

KoRnBlith, Miriam. Venezuela: polarización, revocatoria y después. En WelP, Yanina y SERDÜLT, Uwe. La dosis hace el veneno. Análisis de la revocatoria del mandato en América 
Latina, Estados Unidos y Suiza. Quito, Consejo Nacional Electoral - Instituto de la Democracia, 2014.

LAMBERT, Jacques. América Latina. Barcelona: Ariel, 1974.

LISSIDINI, Alicia. Democracia Directa en Latinoamérica: entre la Delegación y la Participación. En PACHANo, Simón. (Comp.) Temas actuales y tendencias en la ciencia política. Quito: FLACSO Ecuador, 2008.

Martínez Oliveros, Agustina. Los referendos en Venezuela: práctica de la democracia participativa. Cuestiones Políticas, 24, 40, Maracaibo, 2008.

NINO, Carlos. La constitución de la Democracia Deliberativa. Barcelona: Gedisa, 1997.

OCHOA ENRÍQueZ, Haydeé y CHIRINOS ZÁRRAGA, Emilio. Tendencias de la reforma del Estado venezolano en el gobierno de Chávez, Revista Aportes para el Estado y la Administración Gubernamental, 1999.

PAyne, Mark; Zovatto, Daniel; CARrillo, Fernando y Allamand, Andrés. Democracies in Development: Politics and Reform in Latin America. Washington, D.C.: Inter-American Development Bank - International Institute for Democracy and Electoral Assistance, 2002.

RosPigliosi, Fernando. Perú: Elecciones entre 1992 y 1993. En Rial, Juan y ZovaTTO, Daniel. (Eds.) Elecciones y Democracia en América Latina 1992-1996: Urnas y Desencanto Político. San José de Costa Rica: Instituto Interamericano de Derechos Humanos, 1998.

SCHEDLER, Andreas. Conceptualizing accountability- En: SCHEDLER, Andreas; DIAMOND, Larry y PlATTNER, Marc. The self-refraining State: power and accountability in new democracies. London: Lynne Rienner Publishers, 1999.

SERRAFERO, Mario Daniel. Presidencialismo y parlamentarismo en América Latina: un debate abierto, Revista Mexicana de Sociología, Instituto de Investigaciones Sociales, México, 60, 2, pp. 165-186, 1998.

Tuesta SoldeVILla, Fernando. Perú: entre la participación y la gobernabilidad local (19972013). En: WeLP, Yanina y SERDÜLT, Uwe. La dosis hace el veneno. Análisis de la revocatoria del mandato en América Latina, Estados Unidos y Suiza. Quito: Consejo Nacional Electoral Instituto de la Democracia, 2014.

VERdugo Silva, Julio. "Bolivia: entre la expectativa de uso y los intentos fallidos de activaciónEn: WelP, Yanina y SERDÜLt, Uwe. La dosis hace el veneno. Análisis de la revocatoria del mandato en América Latina, Estados Unidos y Suiza. Quito: Consejo Nacional Electoral - Instituto de la Democracia, 2014. 